\title{
EFFECT OF TIME ELAPSED BETWEEN MIXING AND PHOTOACTIVATION ON DEGREE OF CONVERSION, WATER SORPTION AND WATER SOLUBILITY OF SELF-ADHESIVE RESIN CEMENTS
}

\author{
Doaa M. Aboul- Azm D ${ }^{I} B D S$, Ibrahim L. Elgayar ${ }^{2} P h D$, Fayza H. Al-Abbassy ${ }^{3} P h D$
}

\begin{abstract}
INTRODUCTION: Self-adhesive resin cements are designed to adhere to tooth structures without separate adhesive or etchant. The major benefit of these materials would appear to be simplicity of applications with the goal of optimizing adhesive cementation procedures, selfadhesive resin cements were developed to simplify the technique and reduce the possibility of failure on the part of the operator. Low degree of conversion (DC), water sorption and solubility can compromise the mechanical properties and longevity of restorations (1-6).

OBJECTIVES: Is to determine the influence of the photoactivation protocol on the degree of conversion, sorption and solubility in water of two different types of self-adhesive resin cements.

MATERIALS AND METHODS: Two different types of self-adhesive, dual cure resin cements were tested: TEGDMA (Tri-ethylene glycol di-methacrylate) based resin cement [RelyXU200 (RU), 3M ESPE] and UDMA (Urethane di- methacrylate) based resin cement [SmartCem ${ }^{\mathrm{TM}} 2$ (SC2), Dentsply Caulk]. A total of one hundred and twenty cured disc shaped resin cement specimens were prepared for this study using a split teflon mold to be tested for degree of conversion, water sorption and solubility. The specimens were divided into two groups (n=60) according to the type of the cement used, group A was prepared from RU and group B was prepared from SC2. Each group was divided according to the time elapsed between handling of the resin cement and photo-activation into three subgroups $(1,2$ and 3$)$ where $n=20$. Half of each subgroup specimens $(\mathrm{n}=10)$ were used for degree of conversion test while the other half was used for water sorption and solubility test [subgroups A1 \& B1 were immediately photo-activated, subgroups A2 \& B2 were photo-activated 1 minute after handling the cement and subgroups A3 \& B3 were photo-activated 2 minutes after handling the cement]. Ceramic disc (1.5 $\mathrm{mm}$ thickness and $10 \mathrm{~mm}$ diameter) was fabricated using copper split mold to simulate clinical condition. The specimens were irradiated for 40 seconds using LED light applied perpendicular to the specimens at zero distance between the tip of the light cure unit and the ceramic disc. Fourier transformer infrared spectroscopy (FTIR) was used to determine the degree of conversion. For the water sorption and solubility tests specimens were stored in water bath containing distilled water at $37^{\circ} \mathrm{C}$ for 90 days. Data were analyzed using IBM SPSS software package version 20.0

RESULTS: UDMA based cements showed statistically lower degree of conversion for all subgroups compared to TEGDMA based cements which showed significantly higher water sorption than the UDMA based that photo activated after 2 minutes.

CONCLUSIONS: The time interval between mixing and photo activation can significantly influence the properties of the cements and modify their behavior.

KEYWORDS: Self-adhesive resin, degree of conversion, water sorption, water solubility.

1- Bachelor in dentistry, Faculty of Dentistry, University of Alexandria, Alexandria, Egypt.

2- Professor of operative dentistry, Faculty of Dentistry, University of Alexandria, Alexandria, Egypt.

3- Professor of dental biomaterials, Head of Dental Biomaterials Department, Faculty of Dentistry, University of Alexandria, Alexandria, Egypt.
\end{abstract}

\section{INTRODUCTION}

Self- adhesive resin cements have been introduced as onecomponent "universal adhesive cements". They are based on filled polymers designed to adhere to tooth structures and porcelains without the requirement of a separate adhesive or etchant. These cements contain phosphoric acid, which is grafted into the resin. Once mixing is initiated, the phosphoric acid reacts with filler particles and dentin in the presence ofwater forming a bond. They offered dentists a new tool for cementation that had greater ease of use than the existing materials at the time. The monomer's degree of conversion is an important aspect that should be considered as it affects the physicochemical properties including: strength, stiffness, water sorption, solubility, biocompatibility and color stability. Post-operative sensitivity also has been reported as a possible consequence of inadequate polymerization of the resin cements (7). The use of self-adhesive, dual-curing resin cements intends to combine chemical and light polymerization which allowing polymerization to take place in deeper areas of the tooth cavity.

The activation mode may be decisive for the degree of conversion of these dual resin cements and thus on their final properties (6). Furthermore, the final degree of conversion also depends on the chemical characteristics of the monomers present in the formulation (8). Any changes in the chemical structure of the polymeric network formed can cause degradation by hydrolysis (9).

Solubility and water sorption are important features in assessing the clinical durability of dental cements. Consequently, solubility of dental cements has been widely evaluated both in vitro and in vivo (10). Water sorption and solubility may cause degradation of the cements, leading to debonding of the restoration and recurrent decay (5). It could be expected that increasing the time between handling the material and photo activation procedure could stimulate the chemical activation of the material.

On the other hand, an increased viscosity of the material could restrict the photo activation process of polymerization $(11,12)$.

This study aimed to determine the influence of the photo activation protocol on the degree of conversion, water sorption and solubility of self-adhesive, dual-cure resin-based cements. 


\section{MATERIALS AND METHODS}

Two types of self-adhesive, dual-cure resin cements were tested in this study:

-TEGDMA based [Rely X U200 (RU), 3MESPE]. - UDMA based [SmartCem2 (SC2), Dentsply Caulk].

A total of one hundred and twenty cured disc shaped resin cement specimens were prepared for this study (figure1). The cement discs were divided into 2 groups (A and $\mathrm{B}$ ) according to the type of cement under investigation $(n=60)$, group A prepared from TEGDMA based resin cement and group B prepared from UDMA based resin cement. Each group was subdivided according to time intervals between handling and photo activation of resin cements into three subgroups $(n=20)$ where $A 1, A 2$ and $A 3$ are subgroups for group A and B1, B2 and B3 are subgroups for group B.

-Subgroups A1 and B1 were photo-activated immediately after handling the cement.

- Subgroups A2 and B2 were photo-activated 1 minute after handling the cement.

- Subgroups A3 and B3 were photo-activated 2 minutes after handling the cement.Half of the specimens of each subgroup $(n=10)$ were tested for degree of conversion while the other half $(n=10)$ were tested for water sorption and solubility. Each resin cement was mixed according to the manufactures' instructions as following:

-Rely X U200 introduced in the form of clicker so two equal doses of base and catalyst were extruded by the clicker dispenser and were mixed on mixing pad using stainless steel spatula till the base and catalyst were thoroughly blended.

-Smart Cem 2 was mixed and dispensed using the automix syringe.The freshly mixed resin cement was introduced into the mold using teflon coated plastic filling instrument for RelyXU 200, while Smart Cem2 were introduced directly from the mixing syringe into the mold. The material was carefully condensed in the mold cavity using teflon coated plastic filling instrument to be leveled with the upper border of the mold cavity. Care was taken to avoid air entrapment within the specimens, after filling the mold cavity, the Myler strip was placed on the cement and IPS e-max press ceramic disc ( $1.5 \mathrm{~mm}$ thickness and $10 \mathrm{~mm}$ diameter) was placed on the Myler strip in order to simulate the clinical condition as the resin cements under investigation were photo activated through the ceramic disc.

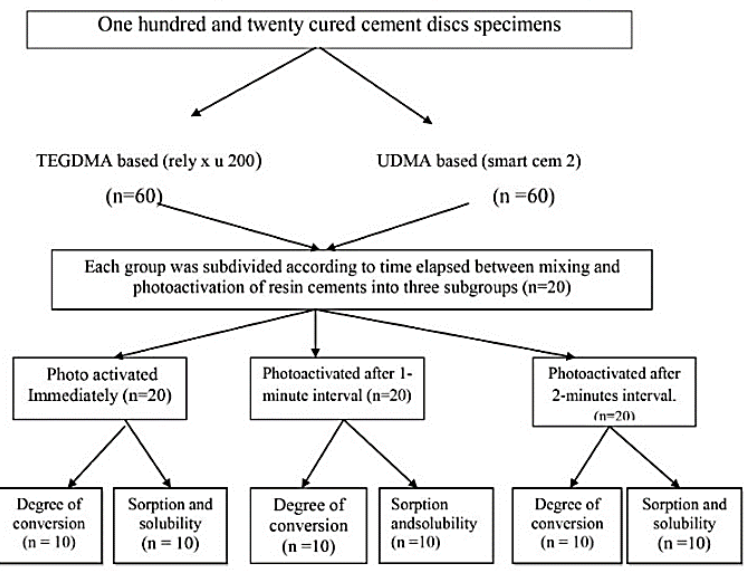

Figure (1): Flow Chart of the study design.

\section{Degree of conversion test}

Fourier transformer infrared spectroscopy (FTIR-8400S, SHIMADZU, Spectrometer,
Japan) was used to determine the degree of conversion of the two types of resin cements under investigation.

Sixty disc-shaped cement specimens $(0.3 \mathrm{~mm}$ thickness and $6 \mathrm{~mm}$ diameter), thirty from each material (group A and B) were prepared using a split teflon mold. The cement specimens from each group were subdivided into three subgroups (A1, A2, A3 and B1, B2, B3) and were photo activated using LED curing unit following the activation protocol times of this study:

1- A1 and B1: Immediate photo-activation after handling the cement $(\mathrm{n}=10)$

2- A2 and B2: Photo activation 1 minute after handling the cement $(\mathrm{n}=10)$.

3- A3 and B3: Photo activation 2 minutes after handling the cement $(\mathrm{n}=10)$.

Immediately, after finishing of the resin cement discs, they were placed in a dry incubator for 24 hours at $37^{\circ} \mathrm{C}$. After the incubation period and before FTIR testing, morter and pestle were used to produce fine powder from each disc individually. Four $\mathrm{mg}$ of resin cement fine powder were mixed with $196 \mathrm{mg}$ pure potassium bromide $(\mathrm{KBr})$ powder for 2 minutes. The mixture was placed under hydraulic pressure $80 \mathrm{KN}$ ( 8 ton) for 5 minutes, and then the pressure evacuated producing transparent discs of $13 \mathrm{~mm}$ diameter, then they were placed in specimen's tray and inserted in the FTIR spectroscopy. For the analysis of the FTIR spectra, the interval between 1800 and $1600 \mathrm{~cm}^{-1}$ was used to observe the signals in 1608 and $1637 \mathrm{~cm}^{-1}$, corresponding to the aromatic and aliphatic vinyl links $(\mathrm{C}=\mathrm{C})$ of the methacrylate functional group, respectively. The degree of conversion of each material was calculated using the ratio between the signal heights of the $\mathrm{C}=\mathrm{C}$ peaks of the polymerized and non -polymerized specimen (13).

DC\% $=\left(1-\frac{\left(1637 \mathrm{~cm}^{-1} / 1608 \mathrm{~cm}^{-1}\right) \text { peak area after curing }}{\left(1637 \mathrm{~cm}^{-1} / 1608 \mathrm{~cm}^{-1}\right) \text { peak area before curing }}\right) \times 100$

Sorption and solubility in water

Teflon split mold with $1 \mathrm{~mm}$ thickness and $6 \mathrm{~mm}$ diameter was used to prepare sixty disc-shaped resin cement specimens, 30 specimens from each cement $(\mathrm{n}=30)$ (according to ISO 4049 standards) $(14,15)$. Group A: TEGDMA based cement $(n=30)$ and group B: UDMA based cement $(n=30)$. As mentioned before, each group was divided into three subgroups according to time elapsed between handling and photo activation of resin cements $(\mathrm{n}=10)$. The specimens were individually dried, stored and kept at room temperature $\left(25 \pm 1^{0} \mathrm{C}\right)$ in the darkness for 24 hours. After this period, they were placed in a desiccator containing desiccants silica gel for 24 hours, as it absorbs water from the specimens. The specimens were then weighed using an analytical balance with a precision of $0.0001 \mathrm{~g}$. This procedure was repeated until a constant weight of $\pm 0.5 \mathrm{mg}$ was obtained ( $\mathbf{m 1}$ ). The specimens were then placed vertically into individual cells (small test tubes) in a water bath containing deionized water for 90 days at a constant temperature of $37^{\circ} \mathrm{C}$. The water was changed weekly. After this period; the specimens were dried with tissue paper and weighed again, thereby obtaining mass (m2). The specimens were again submitted to the previous desiccation for three days and weighed again thereby obtaining mass ( $\mathbf{m} 3)$. The water sorption (Wsp) and water solubility (Wsl) percentages were calculated using the formulae below:

$\% \mathbf{W s p}=\frac{\mathrm{m} 2-\mathrm{m} 1}{\mathrm{~m} 1} \times 100$ 
$\% \mathbf{W s l}=\frac{\mathrm{m} 1-\mathrm{m} 3}{\mathrm{~m} 1} \quad \mathrm{X} 100$

\section{STATISTICAL ANALYSIS}

Data were fed to the computer and analyzed using IBM SPSS software package version 20.0 with significance of the obtained results was judged at the 5\% level. Quantitative data were described using range (minimum and maximum), mean, standard deviation and median. The distributions of quantitative variables were tested for normality using Kolmogorov-Smirnov test, Shapiro-Wilk test and D'Agstino test, also Histogram and QQ plot were used for vision test. The results of the degree of conversion, the sorption and solubility in water results were submitted to two-way ANOVA (material and time between handling and photo activation) and the post hoc Tukey test $(95 \%$ significance).

Kruskal Wallis test was used to compare between different groups. Statistical significance at $\mathrm{P} \leq 0.05$.

\section{RESULTS}

\section{Degree of conversion test (DC)}

There was no statistical statistically significant difference among the three subgroups of TEGDMA based resin cement $(p=0.306)$ and also among the three subgroups of UDMA based resin cement $(p=0.202)$. There was a statistically significant difference between the two types of resin cements (table 1 and figure 2). UDMA based cement (group B) showed statistically lower degree of conversion for all its subgroups compared to the TEGDMA based cement (group A) where $\mathrm{p}=0.002$.

Table (1): Comparison the degree of conversion (DC) values ofthe three studied subgroups according to the type of resin cement.

\begin{tabular}{|c|c|c|c|c|}
\hline & \multicolumn{2}{|c|}{ Degree of conversion (DC) test } & \multirow[b]{2}{*}{$\mathbf{t}$} & \multirow[b]{2}{*}{$\mathbf{P}$} \\
\hline & $\begin{array}{c}\text { Rely X U } 200 \\
\text { (gp. A) }(\mathrm{n}=10)\end{array}$ & $\begin{array}{c}\text { Smart Cem2 } \\
\text { (gp. B) }(\mathrm{n}=10)\end{array}$ & & \\
\hline Immediate photo activation & (gp. $\left.\mathbf{A}_{1}\right)$ & (gp. $\left.B_{1}\right)$ & \multirow{4}{*}{$13.913^{*}$} & \multirow{4}{*}{$<0.001^{*}$} \\
\hline Min. - Max. & $44.0-60.30$ & $29.70-33.0$ & & \\
\hline Mean \pm SD. & $54.70 \pm 4.88$ & $31.37 \pm 1.21$ & & \\
\hline Median & 55.0 & 31.60 & & \\
\hline After 1minute & (gp. $\left.\mathbf{A}_{2}\right)$ & (gp. $\left.B_{2}\right)$ & \multirow{4}{*}{$8.491^{\circ}$} & \multirow{4}{*}{$<0.001^{+}$} \\
\hline Min. - Max. & $49.0-61.0$ & $22.0-38.80$ & & \\
\hline Mean \pm SD & $56.80 \pm 4.35$ & $34.04 \pm 6.76$ & & \\
\hline Median & 56.80 & 37.80 & & \\
\hline After 2minutes & (gp. $\mathbf{A}_{3}$ ) & (gp. $\left.\mathbf{B}_{3}\right)$ & \multirow{4}{*}{$5.744^{*}$} & \multirow{4}{*}{$0.002^{*}$} \\
\hline Min. - Max. & $43.0-63.0$ & $23.0-43.70$ & & \\
\hline Mean \pm SD & $53.27 \pm 5.07$ & $36.38 \pm 7.22$ & & \\
\hline Median & 53.0 & 37.90 & & \\
\hline
\end{tabular}

\section{Water sorption test $\left(\mathbf{w}_{\text {sp }}\right)$}

The water sorption values were higher with longer time intervals for group A (TEGDMA based cement) compared with the group B (UDMA based cement) which did not show any significant differences among its three subgroups $(\mathrm{p}=0.321)$.

The mean values of water sorption and their standard deviations for the three studied subgroups according to the type of resin cement (cement factor) group A and group B are presented in table (2) and figure (3). Group A showed significantly higher water sorption than group B which photo activated after 2 minutes $(p=0.004)$; however, there were no significant differences for immediate photoactivation $(\mathrm{p}=0.445)$ and photoactivation after 1minute $(\mathrm{p}=0.009)$.
Table (2): Comparison the water sorption values of the three studied subgroups according to type of resin cement.

\begin{tabular}{|c|c|c|c|c|}
\hline & \multicolumn{2}{|c|}{ Water sorption test } & \multirow[b]{2}{*}{$\mathbf{Z}$} & \multirow[b]{2}{*}{$\mathbf{P}$} \\
\hline & $\begin{array}{c}\text { Rely X U } 200 \\
(\mathrm{n}=10)\end{array}$ & $\begin{array}{c}\text { Smart Cem2 } \\
(\mathrm{n}=10)\end{array}$ & & \\
\hline Immediate photo activation & (gp. $A_{1}$ ) & (gp. $\left.B_{1}\right)$ & & \\
\hline Min. - Max. & $0.60-3.90$ & $0.66-1.80$ & & \\
\hline Mean \pm SD. & $1.84 \pm 1.22$ & $1.40 \pm 0.44$ & 0.764 & 0.445 \\
\hline Median & 1.80 & 1.60 & & \\
\hline After 1 minute & (gp. $A_{2}$ ) & (gp. $\left.B_{2}\right)$ & & \\
\hline Min. - Max. & $1.14-4.20$ & $0.15-3.0$ & & \\
\hline Mean \pm SD. & $2.73 \pm 0.83$ & $1.62 \pm 0.86$ & $2.619^{*}$ & $0.009^{*}$ \\
\hline Median & 2.70 & 1.95 & & \\
\hline After 2 minutes & (gp. $\left.A_{3}\right)$ & $\left(\right.$ gp. $\left.B_{3}\right)$ & & \\
\hline Min. - Max. & $1.20-4.40$ & $1.0-1.90$ & & \\
\hline Mean \pm SD & $2.92 \pm 1.03$ & $1.56 \pm 0.33$ & $2.881^{\circ}$ & $0.004^{*}$ \\
\hline Median & 3.10 & 1.70 & & \\
\hline
\end{tabular}

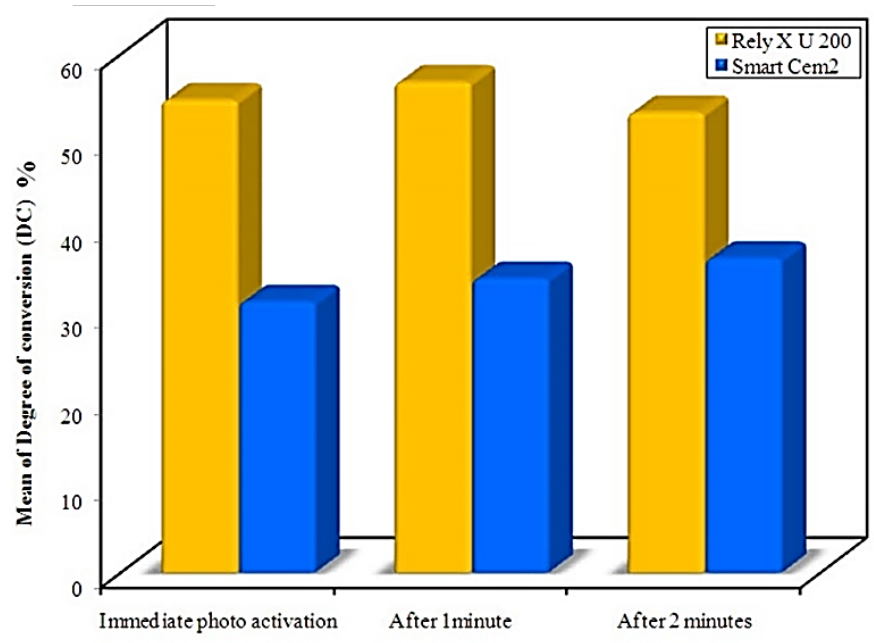

Figure (2): Comparison the degree of conversion (DC) of three studied subgroups according to the type of resin cement.

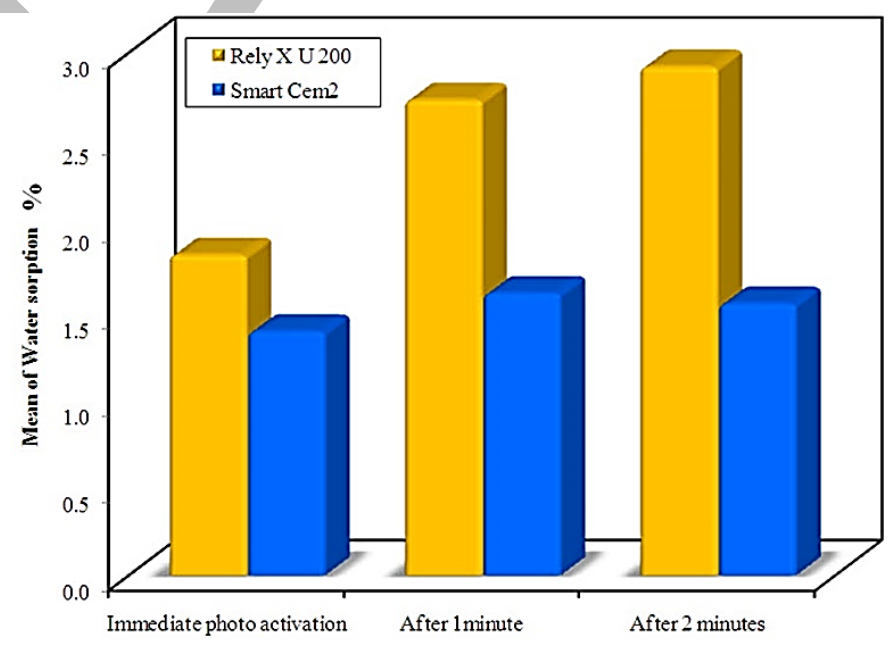

Figure (3): Comparison the values of water sorption of three studied subgroups for Rely X U 200 and Smart Cem2 resin cements.

\section{Water solubility test (wsl)}

The three subgroups of group $A(p=0.879)$ and group $B$ $(p=0.658)$, individually did not present any statistical differences for the different photoactivation times intervals. When comparing the values of water solubility of three studied subgroups according to the type of resin cement (cement factor), which are represented in table (3) and figure (4), the group B had higher solubility than group A for all subgroups. There was no statistical significant 
difference between subgroups of group A and subgroups of group B immediately photoactivated $(p=0.054)$ or photoactivated after $1 \mathrm{~min}$. $(p=0.064)$ but there was a significant difference among the subgroups of both cements photo-activated after 2 minutes $(\mathrm{p}=0.031)$.

Table (3): Comparison the water solubility values for the three studied sub-groups according to type of resin cement

\begin{tabular}{|c|c|c|c|c|}
\hline & \multicolumn{2}{|c|}{ Water solubility test } & \multirow[b]{2}{*}{$\mathbf{Z}$} & \multirow[b]{2}{*}{$\mathbf{P}$} \\
\hline & $\begin{array}{c}\text { Rely X U } 200 \\
(\text { gp. A) }(\mathrm{n}=10)\end{array}$ & $\begin{array}{c}\text { Smart Cem2 } \\
\text { (gp. B) }(\mathrm{n}=10)\end{array}$ & & \\
\hline \multirow{4}{*}{$\begin{array}{r}\text { Immediate photo activation } \\
\text { Min. - Max. } \\
\text { Mean } \pm \text { SD. } \\
\text { Median }\end{array}$} & (gp. $\left.A_{1}\right)$ & (gp. $B_{1}$ ) & \multirow{4}{*}{1.931} & \multirow{4}{*}{0.054} \\
\hline & $0.15-2.0$ & $1.10-2.60$ & & \\
\hline & $1.17 \pm 0.64$ & $1.82 \pm 0.56$ & & \\
\hline & 1.35 & 1.90 & & \\
\hline Afterlminute & (gp. $\mathbf{A}_{2}$ ) & (gp. $\mathrm{B}_{2}$ ) & \multirow{4}{*}{1.856} & \multirow{4}{*}{0.064} \\
\hline Min. - Max. & $0.30-2.70$ & $1.0-3.0$ & & \\
\hline Mean \pm SD & $1.25 \pm 0.92$ & $1.92 \pm 0.74$ & & \\
\hline Median & 1.0 & 1.95 & & \\
\hline After 2minutes & (gp. $\left.A_{3}\right)$ & (gp. $\left.\mathrm{B}_{3}\right)$ & \multirow{4}{*}{$2.159^{*}$} & \multirow{4}{*}{$0.031^{*}$} \\
\hline Min. - Max. & $0.30-2.20$ & $0.80-3.0$ & & \\
\hline Mean \pm SD & $1.06 \pm 0.60$ & $1.71 \pm 0.66$ & & \\
\hline Median & 1.15 & 1.70 & & \\
\hline
\end{tabular}

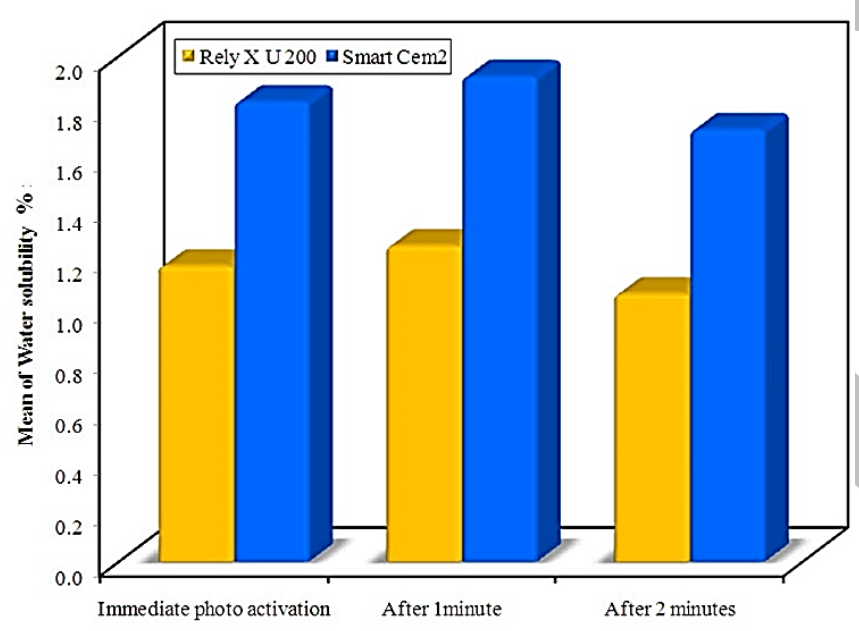

Figure (4): Comparison of water solubility values of the three studied subgroups of Rely X U 200 and Smart Cem2 resin cements.

\section{DISCUSSION}

Resin cements are characterized by simplicity of application, superior mechanical properties, adhesion and esthetic qualities so studying their physical properties may help to predict their ultimate performance.

The specific aim of this study was to investigate the influence of delayed light activation on degree of conversion, water sorption and solubility of two different types of selfadhesive, dual-cureresin cements. It could be expected that increasing the time between handling the material and the photoactivation procedure could stimulate the chemical activation of the material and increase the viscosity of the material. On the other hand, an increased viscosity could restrict the photo activation process of polymerization (11).

\section{Degree of conversion (DC)}

Achieving maximum degree of conversion was always a major concern. Mechanical properties and biocompatibility are highly affected by percentage of unreacted monomers. Decreasing the extent of unreacted monomers in the system reduce the plasticizing effect of these monomers and increase the cross-linking which increase the strength of polymer (16).
Polymerization of the material is determined by the degree of conversion of monomers into polymers. Ideally the dental resin would have all of its monomers converted into polymer during the polymerization reaction; however, all of the dimethacrylate monomers exhibit considerable instauration in the final product with degree of conversion ranging from 55\% to $75 \%$ under normal conditions (17).

Inadequate polymerization may increase residual monomer that could compromise biocompatibility and result in inferior mechanical and physical properties, the polymerization process in dental resins is never complete, and unreacted monomers can remain in the resins after the curing process (18).

According to the literature, higher conversion values are obtained for dual-curing resin cements when the resins are irradiated with high doses of energy while a chemical activation alone presents a significantly lower degree of conversion (19).

In 2009 Arrais et al (20) found that the DC values of autopolymerized resin luting materials were lower than those of the dual-polymerized.

In this study the final conversion of TEGDMA-based (group A) resin cements in 1 and 2 minutes intervals as well as for the UDMA-based resin cements (group B) in1 minute interval did not show any significant change in this parameter; however, TEGDMA-based resin cements (group A) showed a significant decrease in the degree of conversion with the 2 minutes interval. This is probably due to the chemical initiators starting the conversion and increasing the viscosity of the material and thus reduced the mobility of the monomers. This increasing in viscosity prevents the photo activation process from effectively participating in the conversion due to the advanced process of vitrification. This vitrification process occurs during polymerization of composite resins and resinous cements and causes a rapid increase in the modulus of elasticity of the material, which then becomes too rigid to allow plastic flow and free movement of their constituent components (21). In 2013 Marina Di Francescantonio et al (22) found that the DC of resin cements was higher for the low viscosity version, following the light-polymerization.

In this study the group B showed a lower degree of conversion for all groups than the group A. Suitable photo activation procedures prove necessary for dual cements to have higher conversion values and thus obtain better properties.

The use of a ceramic disc on the specimens is a factor of substantial importance, since it mimics the real situation in which the cements are applied as the ceramic restoration causes attenuation in light intensity, thereby reducing the amount of photons reaching the resinous cement and consequently decreasing its conversion (23).

In current study the LED light was used for curing of the resin cements with claimed advantages of light production with a narrow band of wave length (450-490 nm) which situated in the absorption spectrum of champhorquinone photointiator which is $470 \mathrm{~nm}$ (24). So it would be expected that the LED light would provide greater depth of cure (25). In 2005 Bala et al (26) suggested that LED light curing unit produced higher degree of conversion values than halogen light.

\section{Water sorption (Wsp)}

Sorbed water is known to result in internal strains, facilitating the extraction of free monomers or 
polymerization residues in resin-based materials (27). Water is capable of diffusing through polymers, which sorbs water to different degree depending on their molecular and micro structural characteristics.

Water diffuses into the resinous materials and accumulates at the interface between the resin and filler material, reacting with the silane coupler and filler material to release degradation products into the solution (28).

Resin materials are susceptible to water sorption, which is a passive phenomenon of controlled diffusion that occurs gradually and can lead to alterations of the structure and properties of these materials (29).

\section{Water solubility (Wsl)}

Sorption of liquids by resins leads to another phenomenon called solubility. This occurs when unreacted monomers, such as ions and charged particles present in the composition, are leached and consequently cause a weight loss (30).

Cement polymerization in a teflon mold does not have contact with the tooth surfaces and consequently no contact with hydroxyapatites, which neutralize the reaction of the acidic monomers of the cement compositions with their hydroxyl terminals. In this situation some of these multifunctional monomers will not have reacted, and this changes the chemical reaction environment, suitable for the reaction displacement toward product formation. In addition to changing the chemical environment, which is important during an acid-base reaction, as it governs the reaction between the acidic monomers and the inorganic components of the tooth structure, the acidic medium can consume the co-initiators consisting of amines (31). However, RelyXU200 (group A) has sodium sulfonate salts in its composition, potentially preventing chemical incompatibility among the acidic monomers and the chemical initiators, thus preventing a reduction in the degree of conversion of this cement. On the other hand, the difference in sorption could originate from the residual monomers that did not react, due to the absence of a tooth structure and to the water that was not reused for the reaction between the hydroxyapatites and these monomers. Unlike RelyX U200, the time interval produced did not affect SmartCem2 (group B) in terms of water sorption, even though the 2 minutes interval group presented a lesser degree of conversion. This fact is probably due to the difference between the mechanism and the reactional kinetics of these two cements.

When comparing the results for water sorption between the cement groups, the water sorbed by the RelyXU200 cement increased progressively with the time interval; however, the only statistically significant difference was for RelyXU200 two minutes interval, which had the highest water sorption value.

In term of solubility, there was no statistically significant difference between the TEGDA based cement (RelyXU200) groups related to the different time intervals. This may mean that the sorbed water reacted with the components of the material or was imprisoned and became part of the structure. The solubility of the UDMA based cement (SmartCem 2 cement) was not affected by the time interval and the solubility values were compatible with sorption; however, the solubility values of SmartCem 2 were significantly higher than those of RelyXU200.

The studied cements (RelyXU200, SmartCem2) were differently affected by the time interval. This is certainly related to their chemical composition. The 2 minutes interval between manipulation and photoactivation appeared potentially harmful to the clinical performance of both cements evaluated.

Finally, it can be confirmed that the tested hypotheses were rejected, because the delay time before the photo activation of resinous cements influenced the values of conversion, water sorption, and solubility. Moreover, it was evident that the results obtained were dependent on the material in question. In 2014 Silva Fonseca et al (32) evaluated the degree of conversion, sorption, and solubility in water of self-adhesive resin cements subjected to different time interval between material preparation and the photoactivation procedure. They found that the time interval significantly influenced the degree of conversion and water sorption of the resin-based cements. The limitations of this study include:

(1) The fact that commercially available materials were used and, consequently, it is not possible to fully compare the different categories of the materials due to the fact that the materials do not only differ in the organic matrixes, but also in the inorganic, photoinitiator, etc. contents.

(2) The fact that the degradative procedures did not include oral wear simulation.

\section{CONCLUSIONS}

Within the limits of this in vitro study, the following conclusions could be drawn:

1. The time interval between handling and photoactivation can significantly influence the properties of the cements studied and thus, modify their behavior, depending on the material in question.

2. The 2 minutes interval between handling and photoactivation negatively impacted the degree of conversion of RelyX U 200 cement.

3. Solubility of the two cements was not affected by time interval.

4. Solubility values of Smart Cem2 were significantly higher than those of RelyX U200.

\section{CONFLICT OF INTEREST:}

The authors declare that they have no conflicts of interest.

\section{REFERENCES}

1. Sigemori RM, Reis AF, Giannini M, Paulillo LA. Curing depth of a resin modified glass ionomer and two resin-based luting agents. Oper Dent.2005; 30: 185-9.

2. Arrais CA, Rueggeberg FA, Waller JL, de Goes MF, Giannini M. Effect of curing mode on the polymerization characteristics of dual-cured resin cement systems. J Dent.2005; 36: 418-26.

3. Arrais CA, Giannini M, Rueggeberg FA. Effect of sodium sulfinate salts on the polymerization characteristics of dualcured resin cement systems exposed to attenuated lightactivation. J Dent.2009; 37: 219-27.

4. Arrais CA, Giannini M, Rueggeberg FA. Kinetic analysis of monomer conversion in auto- and dual- polymerizing modes of commercial resin luting cements. J Prosthet Dent.2009; 101:128-36.

5. Lüthy H, Filser F, Loeffel O, Schumacher M, Gauckler LJ, Hặmmerle CH. Strength and reliability of four unit allceramic posterior bridges. Dent Mater 2005; 21: 930-7. 
6. Seghi RR, Denry IL, Rosenstiel SF. Relative fracture toughness and hardness of new dental ceramics. J Prosthet Dent1995;74: 145-50.

7. Rosenstiel SF, Land MF and Crispin BJ. Dental luting agents: A review of the current literature. The Journal of Prosthetic Dentistry1998; 80: 280-301.

8. Lang LA, Caputo AA. Flexural strength of a layered zirconia and porcelain dental all-ceramic system. J Prosthet Dent 2005; 94: 125-31.

9. Aboushelib MN, Kleverlaan CJ, Feilzer AJ. Effect of zirconia type on its bond strength with different veneer ceramics. J Prosthod 2008; 17: 401-8.

10. Al-Wahadni A. The roots of dental porcelain: A brief historical perspective. Dental News1999; 2: 43-4.

11. Drummond JL, Eliades G. Ceramic behavior under different environmental and loading conditions. Dental materials in vivo: Aging and related phenomena. Chicago, IL: Quintessence, 2003; 28: 35-45.

12. Evens AG. Slow crack growth in brittle materials under dynamic loading conditions. Int J Fracture 1974;10: 251-9.

13. ISO - Dentistry. Polymer-based filling, restorative and luting materials. Geneva: ISO 4049. (15.07.2000): 18-21.

14. Vrochari AD ,Eliades G, Hellwig E, Wrbas KT. Water sorption and solubility of four self-etching, selfadhesive resin luting agents.J Adhes Dent. 2010; 12: 3943.

15. Cunha L, Alonso R, SobrinoL, Ferracane J. The effect of modulated photoactivation method on contraction stress, degree of conversion and bond strength of composite restorative. J Dent 2007; 35: 318-24

16. Chen HY, Manhart J, Hickel R, et al. Polymerization contraction stress in light-cured packable composite resins. Dent Mater.2001; 17: 253-59.

17. Uctazli S, Tezvergil A, Lasella LV, Vallittu PK. Degree of conversion of fiber reinforced composite using different light curing sources. Dent Mater 2005; 21: 469-75.

18. Asmussen E, Peutzfeldt A. Influence of two step curing on degree of conversion and softening of adental polymer.Dent Mater 2003; 19: 466-70.

19. Bowen RL. Dental filling material comprising vinyl silane treated fused silica and a binder consisting of the reaction product of bisphenol and glycidyl acrylate. USP.1962; 3: 066, 112.

20. Arrais CA, Giannini M, Rueggeberg FA. Kinetic analysis of monomer conversion in auto- and dual-polymerizing, modes of commercial resin luting cements. J Prosthet Dent. 2009; 101: 128-36.

21. Sakaguchi RL, Douglas WH, Peter MCRB. Curing light performance and polymerization of composite restorative materials. J Dent. 1992; 20: 183-8.

22. Marina Di Francescantonio M, Aguiar TR, Arrais CA, Cavalcanti ANMarina Di Francescantonio M, Aguiar TR, Arrais CA, Cavalcanti AN, Davanzo CU, Giannini M. Influence of viscosity and curing mode on degree of conversion of dual-cured resin cements. Eur J Dent. 2013; 7: $81-5$.

23. Suh BI, Feng L, Pashley DH, et al. Factors contributing to the incompatibility between simplified-step adhesives and chemically-cured or dual-cured composites. Part III. Effect of acidic resin monomers. J Adhes Dent 2003; 5: 267-82.

24. Wiggins KM, Hartung M, Althoff O, Wastian C, Mitra SB. Curing performance of a new generation light emitting diode dental curing unit. J Am Dent Assoc 2004; 135: 14719.
25. Han L, Okamoto A, Fukushima M, et al. Evaluation of physical properties and surface degradation of self-adhesive resin cements. Dent Mater J 2007; 26: 906-14

26. Bala O, Olmez A, Kalyci S. Effect of LED and halogen light curing on polymerization of resin based composites. J Oral Rehabi.2005; 32: 134-40

27. Santos C, Clarke RL, Braden M, Guitian F, Davy KW. Water absorption characteristics of dental composites incorporating hydroxyapatite filler. Biomaterials 2002; 23: 1897-1904.

28. Luci Regina panka Archegas, Rodrigo Nunes Rached, Segrio Aparecdo Ignacid, Elianecarvalho de Vasconcelos, Debora Toledo Ramos. Identification and Qualification of monomer released from dental composite using HPLC. Braz. Arch. Biol. technol. 2009; 52: 855.

29. Ferracane JL. Hygroscopic and hydrolytic effects in dental polymer networks. Dent Mater 2006; 22: 211-22

30. International Organization for Standardization. ISO4049: 2000- Polymer-based filling, restorative and luting materials. Geneva: ISO; 2000; 28

31. Ilie N, Hickel R. Correlation between ceramics translucency and polymerization efficiency through ceramics. Dent Mater 2008; 24: 908-14.

32. Andrea Soares Quirino da Silva Fonseca, Jamil Mizrahi, Livia Rodrigues Menezes, Lisia Lorea Valente, Rafael Ratto de Moraeset al. The effect of time between handling and photoactivation on self-adhesive resin cement properties. J. Prosthodontics. 2014; 23: 302-7. 